\title{
Artefact
}

Techniques, histoire et sciences humaines

$11 \mid 2019$

Pannes et accidents ( $\mathrm{XIX}^{\mathrm{e}}-\mathrm{XX}{ }^{\mathrm{e}}$ siècle)

\section{Autour de l'explosion du navire à vapeur l'Industrie dans le port de Marseille}

Statuts, identité et compétences des mécaniciens de la marine marchande au milieu du XIX ${ }^{\mathrm{e}}$ siècle

About the explosion of the steamer Industry in Marseilles harbour: statutes, identity and skills of the engine drivers in the mid-nineteenth century French merchant navy

\section{Xavier Daumalin et Olivier Raveux}

\section{Q OpenEdition}

\section{Journals}

\section{Édition électronique}

URL : http://journals.openedition.org/artefact/4447

DOI : 10.4000/artefact.4447

ISSN : 2606-9245

Éditeur:

Association Artefact. Techniques histoire et sciences humaines, Presses universitaires du Midi

\section{Édition imprimée}

Date de publication : 15 décembre 2019

Pagination : 19-38

ISBN : 978-2-8107-0667-9

ISSN : 2273-0753

Référence électronique

Xavier Daumalin et Olivier Raveux, "Autour de l'explosion du navire à vapeur l'Industrie dans le port de Marseille », Artefact [En ligne], 11 | 2019, mis en ligne le 27 novembre 2020, consulté le 30 novembre 2020. URL : http://journals.openedition.org/artefact/4447 ; DOI : https://doi.org/10.4000/ artefact. 4447

\section{(ब) $\odot$}

Artefact, Techniques, histoire et sciences humaines est mise à disposition selon les termes de la Licence Creative Commons Attribution - Pas d'Utilisation Commerciale - Pas de Modification 4.0 International. 


\title{
Autour de l'explosion du navire à vapeur l'Industrie dans le port de Marseille
}

\author{
Statuts, identité et compétences des mécaniciens \\ de la marine marchande au milieu du XIX ${ }^{\mathrm{e}}$ siècle \\ Xavier Daumalin et Olivier Raveux
}

\section{Résumé}

À travers létude d'un accident - l'explosion d'une des chaudières du paquebot l'Industrie dans le port de Marseille en décembre 1851 - et de ses conséquences judiciaires, cet article entend examiner les changements provoqués par l'innovation technique dans le monde du travail maritime au cours de la première révolution industrielle. Le propos est centré sur la figure du mécanicien, personnage emblématique de la machine à vapeur. II s'attache tout d'abord à retracer et à expliquer l'évolution des statuts du responsable des machines, notamment ses positions sur le navire et dans les textes réglementaires, deux éléments placés au cœur de l'affaire et dont les tribunaux s'emparent afin de rendre la justice. L'accident de l'Industrie permet également de mener l'enquête sur la question de la construction des compétences et de l'identité du mécanicien. Le deuxième tiers $d u x x^{e}$ siècle apparaît à cet égard comme un moment crucial, qui achève de transformer le métier de mécanicien sur les navires à vapeur en véritable profession maritime.

\section{Mots-clés}

accident, innovation technique, transport maritime, mécanicien, justice, réglementation

95 Xavier Daumalin et Olivier Raveux, « Autour de l'explosion du navire à vapeur l'Industrie dans le port de Marseille », Artefact, 11, 2019, p. 19-38. 


\section{About the explosion of the steamer Industry in Marseilles harbour}

Statutes, identity and skills of the engine drivers in the midnineteenth century French merchant navy

\section{Abstract}

By studying an accident-a boiler explosion of the steamer Industry in Marseilles harbour in December 1851 - and its judicial consequences, this article intends to examine the changes brought by technical innovation in maritime work during the first industrial revolution. The focus is on the figure of the engine driver, the emblematic character of the steam engine. First of all, this work seeks to trace and explain the evolution of the mechanic statutes, in particular his positions on board and in the regulatory texts, two elements placed at the heart of the case and dissected by courts in order to do justice. The accident of the Industry also makes it possible to carry out the investigation on the question of the construction of the skills and the identity of the mechanics. The second third of the nineteenth century appears in this respect as a crucial moment, which completes the transformation of the job of engine driver into a real maritime profession.

\section{Keywords}

accident, technical innovation, shipping, engine driver, justice, regulations

\section{Note des auteurs}

Cette recherche a été financée par le programme ERC 714437 SeaLiT (Seafaring Lives in Transition. Mediterranean Maritime Labour and Shipping during Globalization). 


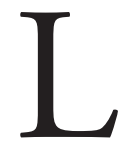
es changements provoqués par l'innovation technique dans le monde du travail en France au cours de la première révolution industrielle mobilisent depuis longtemps les travaux des historiens. Les recherches ont livré d'importants résultats sur des sujets aussi variés que l'emploi, l'organisation de la production, les conditions d'activité, la formation professionnelle ou encore la santé ${ }^{1}$. Cependant, tous les secteurs économiques n'ont pas fait l'objet du même traitement. Alors que l'industrie, les mines et les chemins de fer ont monopolisé l'attention, la marine marchande a plutôt été réduite à la portion congrue. Seuls les travaux de Jean-Louis Lenhof et de Nicolas Cochard ont levé un premier voile sur le sujet ${ }^{2}$. Le dossier des mutations déclenchées dans le travail au sein de cette branche d'activité par l'introduction de la vapeur reste ouvert. Le but de cet article est d'apporter une contribution à ce chantier grâce à une étude partant d'un accident d'exploitation - l'explosion d'une chaudière du paquebot l'Industrie dans le port de Marseille en décembre 1851 - et centrée sur la figure du mécanicien de bord.

Quelles sont les raisons de ces choix ? Des travaux situés au croisement de l'histoire économique, de l'histoire des techniques et de l'histoire sociale ont déjà validé les vertus méthodologiques et la richesse des apports de l'analyse d'événements, comme ceux réalisés, par exemple, par Claire Barillé, Thomas Le Roux et Marie Thébaud-Sorger autour de l'explosion de la poudrerie de Grenelle à Paris en $1794^{3}$. L'historien le sait et en profite : les situations à caractère exceptionnel produisent bien souvent une documentation hors norme, aussi bien en quantité qu'en qualité. Grâce à ce « matelas » archivistique, une micro-histoire d'un accident d'un appareil ou d'un dispositif permet de rentrer au cœur du système technique qui l'abrite et de l'organisation sociale qui en a la charge.

L'entrée par l'accident revêt un autre intérêt : elle permet de déplacer la focale d'observation sur le terrain du juridique et de l'administratif. Dans le cadre des réparations de dommages et de la prévention des risques, les accidents poussent les tribunaux, l'administration et les pouvoirs législatifs

1. Il est impossible de donner ici une véritable bibliographie, même très sélective. Nous nous contenterons de citer, parce qu'il touche à de nombreux sujets et qu'il est emblématique de ce champ de recherche, le travail de François Jarrige : Jarrige, 2009.

2. Entre autres publications, Cochard, 2011 ; Cochard, 2015 ; Barzman, Lenhof (dir.), 2006 ; Lenhof, 2006.

3. Barillé, Le Roux, Thébaud-Sorger, 2014. 
à produire des statuts, à créer des réglementations sur les compétences et à définir les responsabilités des travailleurs en charge des machines. À ce titre, ils forment un laboratoire de tout premier ordre pour saisir les changements provoqués par l'innovation technique dans le monde du travail maritime $e^{4}$. Dans notre étude de cas, c'est la catégorie du personnel rendu responsable de l'accident de l'Industrie, le mécanicien, qui émerge. Apparu sur les navires avec l'introduction de l'énergie vapeur, ce travailleur embarqué garde longtemps des statuts assez flous et peine durant plusieurs décennies à intégrer pleinement le milieu très traditionnel de la marine marchande.

L'analyse sera conduite en trois temps. Le premier sera celui de la narration de l'accident et de l'étude de ses prolongements devant les tribunaux, depuis le premier jugement, celui du tribunal civil de Marseille, jusqu’à l'arrêt de la Cour de cassation, qui met un terme à l'affaire et établit une jurisprudence en matière de responsabilité civile sur les dommages créés par l'explosion d'un appareil à vapeur sur un navire. Le propos se déplacera ensuite sur la lecture et la construction des statuts du mécanicien, notamment ses positions sur le navire et dans les textes réglementaires, deux éléments placés au cœur de l'affaire et dont les tribunaux s'emparent afin de rendre la justice. Enfin, le travail se terminera par une étude sur les compétences et l'identité du responsable de la machine. Le deuxième tiers du XIX ${ }^{\mathrm{e}}$ siècle, période durant laquelle ont lieu l'accident et ses conséquences judiciaires, forme un moment crucial, qui achève de transformer le métier de mécanicien sur les navires à vapeur en véritable profession maritime. Les compétences techniques ne sont plus seulement révélatrices d'un savoir-faire ; elles sont désormais au cœur de la formation d'une activité définie, réglementée et surveillée.

\section{L'accident et ses prolongements judiciaires}

Dès les années 1820, « les chaudières françaises sont normalisées dans leurs paramètres essentiels : épaisseur des tôles, point de fusion des rondelles fusibles et diamètre des soupapes en fonction de la pression d'usage et du

4. Sur la technologie de la vapeur appliquée à la marine marchande durant les deux premiers tiers du XIX ${ }^{\mathrm{e}}$ siècle, on se reportera à Lenhof, 2005. 
diamètre des chaudières $»^{5}$. Ces prescriptions sont utiles pour le traitement des accidents, aussi bien en amont qu'en aval. Elles visent à prévenir les explosions et à identifier, quand les drames n'ont pu être évités, le ou les responsables. Dès lors, l'existence d'une faute et la production d'un fautif permettent à la justice d'attribuer des dédommagements aux victimes (art. 1382 du Code civil de 1804). Mais le responsable des faits n'est pas forcément le responsable civil. Ainsi, dans le cadre d'une entreprise, les liens d'autorité et les statuts des employés sont des éléments à prendre en compte. Qui doit payer au final ? C'est tout l'enjeu des procès provoqués par l'accident de l'Industrie dans le port de Marseille en 1851.

\section{L'explosion de l'Industrie}

Au petit matin du 15 décembre 1851, le paquebot de propulsion mixte l'Industrie se prépare à lever l'ancre dans le bassin de la Joliette à Marseille. Ce vapeur de 120 chevaux de puissance, doté de 22 membres d'équipage, appartenant à la compagnie des frères Valéry et récemment sorti des chantiers navals de La Ciotat s'apprête à effectuer sa liaison habituelle vers Bastia et Livourne ${ }^{6}$. À sept heures, alors que la machine est en chauffe, le capitaine du navire, Érasme Santi, doit momentanément quitter le bord pour aller en ville remplir les obligations imposées par le cahier des charges de la concession postale que sa compagnie détient pour le service de la Corse depuis l'année précédente ${ }^{7}$. Ordre est donné au mécanicien, dont nous ne connaissons pas l'identité, « de ne faire monter la vapeur qu'à neuf heures ". Ses affaires réglées, le capitaine est de retour vers son paquebot sur une chaloupe et aperçoit du milieu du port de la fumée sortir du milieu du navire. Il redoute l'incendie mais apprend, arrivé à bord, que « la chaudière a éclaté dans la partie arrière du coffre ". Le bilan est lourd : plus d'une douzaine de personnes ont été gravement atteintes par des projections de vapeur et d'eau bouillante.

5. Fressoz, 2014, p. 85. On se reportera à cet article sur la question des normes de sécurité et de responsabilité civile à propos des appareils à vapeur.

6. Sur la compagnie corse Valéry frères, $c f$. Alessandri, 1985 ; Finidori, Mambrini, 2009.

7. Informations tirées du rapport de mer après l'accident (AD Bouches-du-Rhône, 531 U 170, rapport du capitaine Érasme Santi du 15 décembre 1851). Les rapports de mer sont rédigés par le capitaine et relatent les événements s'étant déroulés pendant le voyage. Déposés au tribunal de commerce, ils font foi jusqu'à preuve du contraire et constituent un acte juridique essentiel à la défense des intérêts du navire. 


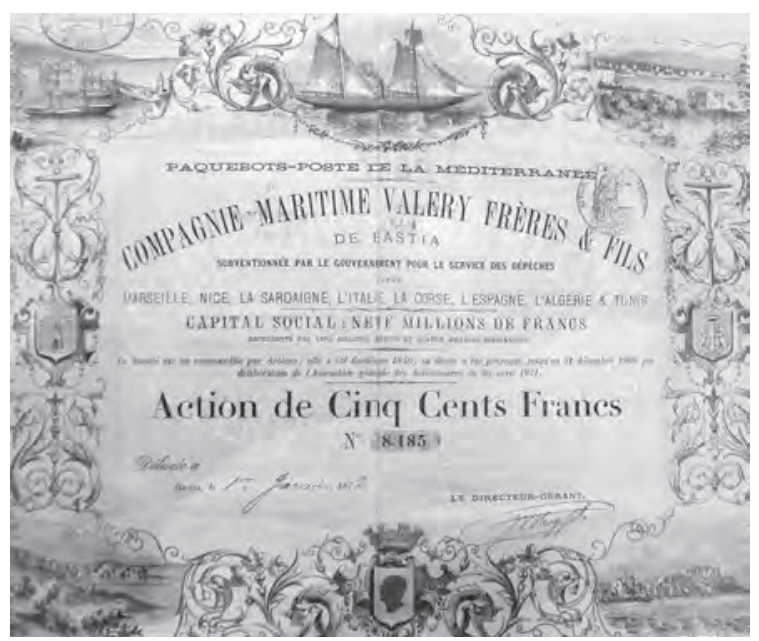

\section{Fig. 1. - Action de la Compagnie Valéry frères \& fils, 1872}

Archives de la chambre de commerce et d'industrie Marseille-Provence.

Cliché Olivier Raveux

Ce type d'accident est-il courant ? Si la période des premiers tâtonnements techniques de la navigation à vapeur et de la crainte des " cratères ambulants $^{8}$ " est révolue, les pannes et les accidents de chaudières restent nombreux sur les steamers et sont quasi endémiques à un système de transport qui doit mener une lutte continue contre les incidents mécaniques? En 1851, les problèmes sont cependant plutôt bénins. Pour l'essentiel, il s'agit de bris de tuyaux d'alimentation, d'échappements de vapeur suite à de petites fissures sur les tôles et de fuites d'eau mineures sur les foyers. Les explosions de chaudière sont devenues rares et " n'ont eu lieu que sur de petits navires ou dans des établissements peu importants dont le personnel assorti aux ressources était loin de satisfaire aux conditions nécessaires ; tandis que les grandes entreprises savent payer de bons mécaniciens et entretenir des appareils d'une grande valeur ${ }^{10}$. La Compagnie Valéry frères vient valider ce constat et l'épisode de l'Industrie n'est d'ailleurs pas

8. C'est ainsi que la population appelle encore les vapeurs de la Compagnie Rostand à Marseille dans les années 1840 : Barak, 1971.

9. À l'image de tous les autres secteurs de l'industrialisation, $c f$. Landes, 1975, p. 125.

10. Bonnefoux et Paris, 1859, p. 300. 
une nouveauté pour les armateurs cap-corsins. Au cours des années précédentes, deux autres navires de cette compagnie ont été victimes d'accidents mortels liés à des problèmes de chaudières. En 1846, un des générateurs de vapeur du Maréchal Sébastiani s'est déchiré sur $60 \mathrm{~cm}$, provoquant la mort du personnel de la machine ${ }^{11}$. En septembre 1847 , les haubans retenant la cheminée du Comte de Paris se rompent alors qu'il fait route vers Marseille, libérant de la vapeur du haut des chaudières dans la salle des machines et tuant trois ouvriers, dont le chef mécanicien Welle ${ }^{12}$. Pour la Compagnie Valéry frères, l'accident de l'Industrie s'inscrit donc dans une série noire et présente de plus une nouveauté inquiétante : pour la première fois des passagers figurent parmi les blessés, ouvrant le risque de demandes de dédommagement devant les tribunaux civils. Et c'est très exactement ce qui se produit. Quelques jours après l'accident, un des passagers blessés, un dénommé Visconti, assigne les frères Valéry devant le tribunal civil de Marseille.

\section{Prolongements devant les tribunaux}

L'enquête sur les causes de l'explosion de la chaudière donne rapidement ses résultats. Selon l'ingénieur en chef des Mines du département des Bouches-du-Rhône, l'accident a été provoqué par deux éléments, un secondaire et un déterminant. La raison mineure est un vice de construction, à savoir un diamètre insuffisant des soupapes de sûreté ${ }^{13}$. La principale explication est une surcharge de vapeur dans l'appareil, dont la faute est imputable au mécanicien et à son subordonné, le chauffeur, qui «ont chauffé outre mesure ${ }^{14}$. Si les fautifs paraissent avoir été trouvés, il faut encore déterminer qui doit prendre en charge les indemnisations. Pour Visconti, c'est à l'armateur qu'il incombe de réparer financièrement. La procédure judiciaire dure presque trois ans. Les juges de différentes instances doivent répondre à une question paraissant simple de prime abord :

11. Finidori, Mambrini, 2009, p. 27-28.

12. Le Sémaphore de Marseille, 19 septembre 1847.

13. Il est à noter, sur la question de la soupape, que la commission de surveillance des machines à vapeur des Bouches-du-Rhône avait visité la chaudière et l'avait déclarée conforme. L'élément n’ayant pas été jugé décisif dans l'explosion du générateur, l'entreprise Louis Benet \& compagnie de La Ciotat n’a pas été inquiétée et les frères Valéry ne la nomment d'ailleurs jamais devant les tribunaux.

14. Sur les différents jugements et l'énoncé des motifs, cf. Recueil général des lois et des arrêts, en matière civile, criminelle, administrative et de droit public, Paris, 1854, p. 236-238. 
qui est responsable civilement des accidents causés par l'explosion de la machine à vapeur d'un navire quand l'événement survient par la faute du mécanicien?

Les frères Valéry se refusent en effet à payer les dédommagements. Leur argumentation réside en un point : " le mécanicien et le chauffeur étaient les préposés du capitaine, dont la responsabilité se trouve dès lors engagée ». Les armateurs cap-corsins s'appuient sur leur lecture de la législation en vigueur. Le Code de commerce de 1807 a confirmé la totale autorité du capitaine sur un navire, "seul maître à bord ". À ce titre, il serait donc responsable civilement des conséquences des actions de tous les membres de l'équipage. L'usage de la vapeur et l'emploi de mécaniciens n'auraient pas changé la donne selon les frères Valéry. Par l'article 41 de l'ordonnance royale du 17 janvier 1846 relative aux bateaux français qui naviguent sur mer, le mécanicien est placé sous l'autorité du capitaine dans toutes ses activités ${ }^{15}$. Les nouveaux éléments de la législation n'auraient donc fait que confirmer, en les étendant aux nouveaux membres de l'équipage, l'ancienne chaîne d'autorité à bord des navires et de responsabilité civile devant les tribunaux.

Le jugement du tribunal civil de Marseille du 8 mars 1853 tranche en faveur de Visconti et condamne les frères Valéry à lui verser un dédommagement de 5500 francs. Les motifs de la décision s'appuient sur une appréciation de l'article 216 du Code de commerce de 1807 : le propriétaire d'un navire est civilement responsable, non seulement des fautes du capitaine, mais aussi de celles que tous les autres membres de l'équipage commettent dans le cadre de leur service. Les frères Valéry font appel devant la Cour impériale d'Aix-en-Provence, devant laquelle ils sont déboutés le 17 août 1853, puis saisissent la Cour de cassation, pour un résultat défavorable annoncé le 29 mars 1854. La chaîne de responsabilité civile est confirmée. En est-il de même pour les liens d'autorité et les statuts à bord du navire? Pas vraiment. Si la décision finale est chaque fois la condamnation des frères Valéry, les chemins argumentatifs utilisés par les différentes instances de justice pour se prononcer ne sont pas identiques.

Le tribunal civil de Marseille a en effet proposé une nouvelle lecture de la chaîne d'autorité à bord des navires. Suivons les motifs du jugement :

15. Sur cette ordonnance, $c f$. Collection complète des lois, décrets, ordonnances, règlements et avis du Conseil d'État, 1846, p. 37-43. 
les deux pouvoirs du capitaine et du mécanicien, quoique inégaux, n’en sont pas moins indépendans et s'appliquent à des objets différens [...] les mécaniciens, soit par la nature, soit par l'importance de leurs fonctions à bord, ne peuvent pas être considérés comme des hommes d'équipage au choix des capitaines; que ce choix, plus important peut-être que celui des capitaines eux-mêmes, est laissé aux soins des propriétaires [...] ; que choisis par les propriétaires, ils engagent la responsabilité de ces derniers comme leurs préposés.

Autrement dit, si l'armateur est bel et bien responsable des faits du mécanicien, ce dernier n'est pas pour autant placé sous la direction du capitaine dans ses missions sur la machine. Cette vision des choses entre en totale contradiction avec l'ordonnance de 1846 et constitue une erreur d'appréciation que la Cour de cassation ne manque d'ailleurs pas de corriger dans son arrêt de mars 1854 .

Concernant le statut du mécanicien, sa position à bord du navire et la spécificité de ses connaissances et de son savoir-faire, ces errements des tribunaux de première instance mettent en lumière une série de questions autour des conséquences de l'introduction de la vapeur dans les caractéristiques et le fonctionnement des équipages. Ses compétences particulières lui donnent-elles, sinon une part d'autorité, tout du moins une marge de manœuvre indépendante de celle du capitaine ? Comment le système social traditionnel du travail maritime et le système technique de la vapeur se sont-ils ajustés pour tenter de produire une organisation du travail cohérente et efficace à bord des navires ?

\section{Les statuts du mécanicien}

L'introduction de la vapeur a instantanément modifié le travail maritime, notamment en faisant naître de nouveaux métiers à bord des navires, depuis les soutiers jusqu'aux mécaniciens, en passant par les graisseurs et les chauffeurs. Au cours des années 1830-1850, quand la navigation est encore dite "mixte ", c'est-à-dire s'appuyant dans le même temps sur le vent et l'énergie vapeur, les personnels de la machine, travaillant dans le "ventre " des bateaux, forment un groupe qui s'oppose spatialement et psychologiquement aux marins du pont supérieur, " dont la place s'est 
trouvée amoindrie par des hommes et des machines devenus désormais indispensables ${ }^{16}$ ». Néanmoins, les mutations hiérarchiques et statutaires relatives à l'impact provoqué par l'apparition et le développement rapide de la nouvelle source d'énergie tardent à se dessiner clairement et cet élément contribue à laisser le mécanicien de bord comme un personnel au mieux sans grande visibilité officielle, au pire en décalage important avec le milieu dans lequel il travaille.

\section{Le mécanicien : un personnel de bord bien singulier}

Nous l'avons vu avec l'arrêt de la Cour de cassation concernant l'accident de l'Industrie : le développement de l'utilisation de l'énergie vapeur n'a pas modifié les dispositions contenues dans le Code de commerce de 1807, ainsi que dans les lois et les textes réglementaires publiés par l'État français jusqu'à la seconde moitié des années 1840. Le capitaine conserve son autorité sur tous les membres de l'équipage. Les hommes de la salle des machines sont-ils pour autant des personnels embarqués comme les autres? Quelles sont les positions qu'ils occupent au sein de l'équipage, dans la chaîne d'autorité sur le navire et dans la législation?

Les soutiers et les simples chauffeurs sont considérés comme des marins et doivent d'ailleurs gagner le pont supérieur à la demande du capitaine, quand il s'agit d'aider en urgence à la manœuvre des voiles. Il en va bien autrement pour le personnel qualifié, chauffeurs-graisseurs et mécaniciens, qui s'inscrivent certes dans la même chaîne hiérarchique, mais qui présentent dans le même temps des caractéristiques singulières. En effet, au cours des premières décennies de la navigation à vapeur, ces hommes ne relèvent pas de l'inscription maritime, le système de recensement des personnes pouvant être appelées à servir sur les bateaux des forces militaires françaises ${ }^{17}$. Ils ne sont donc pas considérés comme des marins et sont d'ailleurs choisis non par le capitaine, comme c'est habituellement l'usage dans la navigation, mais par l'armateur. C'était d'ailleurs le point central des motifs du jugement délivré le 8 mars 1853 par le tribunal civil

16. Cochard, 2011, p. 160. Sur les espaces socio-professionnels du navire et les cultures des différents métiers s'exerçant à bord, Lenhof, 2006.

17. Pawlowski, 1910 et Boyer, 1960. 
de Marseille pour que les frères Valéry assument financièrement les dommages causés par la faute du mécanicien de l'Industrie.

Le fait que le personnel qualifié de la machine ne soit pas compris statutairement dans la catégorie des marins a une traduction très concrète dans la vie et dans le travail à bord des navires. Alors même qu'il porte l'uniforme, signe tangible d'une certaine autorité, et qu'il commande aux travailleurs de la salle des machines, le chef mécanicien est seulement considéré comme un personnel d'entretien et d'exécution, et non comme personnel d'encadrement ${ }^{18}$. Il n'a pas le statut d'officier de bord et reste tenu à l'écart des discussions importantes de l'état-major. Les rapports de mer fournissent de très nombreux exemples de cette mise à distance du mécanicien dans les prises de décision du personnel d'encadrement, y compris dans la gestion des incidents de voyage liés à des problèmes d'appareils à vapeur. Le responsable de la machine "prévient " le capitaine, "donne son avis ", " fait rapport " ou " livre ses observations ${ }^{19}$. La capitaine et les officiers prennent entre eux la décision au final. Au cours des années 1830-1850, tout démontre que les mondes professionnels de la navigation traditionnelle et de la mécanique peinent à travailler en bonne intelligence. D’une manière générale, il faut même qualifier les relations entre les capitaines et les mécaniciens de conflictuelles au cours de cette période.

\section{Du conflit à l'intégration au milieu d'exercice}

À l'image de plusieurs entreprises de navigation à vapeur de cette période, la Compagnie des Messageries impériales ${ }^{20}$ évoque dans son assemblée générale du 28 mai 1853 les conflits incessants qui opposent les mécaniciens aux commandants de bord et explique qu'elle a dû recourir, pour y mettre un terme, à des mesures disciplinaires à l'encontre des premiers

18. Sur la tripartition fonctionnelle des équipages, Lenhof, 2006, p. 78.

19. AD Bouches-du-Rhône, 531 U 170, rapport du capitaine Caboufigue, $1^{\text {er }}$ décembre 1851 ;

531 U 174, rapport du capitaine Lauzet, 23 août 1852 ; 531 U 189, rapport du capitaine Arnaud, 5 janvier 1855 ; 531 U 191, rapports des capitaines Daniel et Bocandé, 9 juin et 11 juillet 1855 ; 531 U 193, rapport du capitaine Bourdeau, 29 décembre 1855 ; 531 U 195, rapport du capitaine de Sardi, 30 avril 1856 ; etc.

20. La Compagnie des Messageries nationales a été créée en 1851 et prend le nom de Messageries impériales en 1853. Sur cette entreprise, Berneron-Couvenhes, 2007. 
nommés ${ }^{21}$. Le cœur des différends réside dans la gestion de tout ce qui touche aux appareils à vapeur, affaires présentées dans les rapports comme des « empiètements dans les affaires de machine». Si les principes de commandement en la matière sont simples en théorie - le capitaine ordonne et le mécanicien exécute -, la mise en pratique s'avère en revanche plus délicate. Le rapport des Messageries impériales de l'exercice 1852 sur le premier mécanicien du Louqsor est ici emblématique pour révéler l'état d'esprit d'hommes fiers de compétences valant parcelle d'autorité : il est « imbu de son savoir » et " animé comme beaucoup d'entr'eux [les mécaniciens] du désir de faire de sa machine un petit gouvernement à part ${ }^{22}$. Ces conflits d'autorité empêchent la création d'un milieu professionnel homogène au service des compagnies de navigation. Les pouvoirs publics tardent à prendre les mesures susceptibles de mettre fin aux problèmes. Dans son article 57, le Code disciplinaire et pénal de la marine marchande du 24 mars 1852 énumérant les fonctions qui donnent droit au titre d'officier à bord des navires de commerce ne fait aucune mention du mécanicien. Un rapport adressé à l'Empereur sur la situation de la marine marchande vient encore souligner en 1864 que les « les lois et décrets [...] n'ont pas défini leur situation [celle des mécaniciens] d'une manière précise $[\ldots]^{23} »$. L'impulsion décisive semble avoir été donnée par les entreprises privées. En 1851, les chefs et les seconds mécaniciens de la Compagnie des Messageries nationales, une des plus grandes sociétés de transport maritime du temps, sont reconnus comme officiers et sous-officiers de bord. L'État finit par emboîter le pas quelques années après, en prenant une série de mesures destinées à mieux intégrer le mécanicien dans son milieu d'exercice, en en faisant un membre à part entière du monde des marins. Par le décret impérial du 28 janvier 1857, tous les hommes employés au service des machines à vapeur dans la marine marchande sont désormais compris dans l'inscription maritime ${ }^{24}$. Il faut encore attendre quelques années supplémentaires pour que les mécaniciens voient leur statut complété, mis en

21. Archives de la Chambre de commerce de Marseille, fonds des Messageries Maritimes, L/19/60/427/1, assemblée générale du 28 mai 1853.

22. Association French Lines, Le Havre, fonds des Messageries Maritimes, 1997-002-0217, dossier de Joseph Fourmigault.

23. Journal du Palais. Lois, décrets, règlements et instructions d'intérêt général. Année 1864, Paris, 1865 , p. 116.

24. La mesure était attendue par la Marine impériale, dans la mesure où les mécaniciens des vaisseaux de guerre étaient jusqu’alors des engagés volontaires ou des recrutés. Par le décret impérial 
conformité avec l'autorité qu'ils détiennent sur le personnel de la machine et les responsabilités qu'ils assument sur les appareils moteurs. Grâce à l'article premier du décret impérial du 21 septembre 1864, "les mécaniciens en chef et ceux chargés en sous-ordre de la direction de la machine ont rang d'officiers à bord des bâtiments à vapeur du commerce ${ }^{25}$ ". Si le mécanicien finit donc par trouver un vrai statut et une vraie place dans le monde du travail maritime, force est de constater que le processus a été lent. La rapidité du développement de l'énergie vapeur n’a pas trouvé d'équivalent dans l'organisation statutaire et hiérarchique du travail à bord des navires, créant un hiatus entre le nouveau système technique et le système socioprofessionnel qui l'utilise.

\section{Identité et compétences du mécanicien}

La mise en place d'un statut intégratif du mécanicien suffit-elle à régler tous les problèmes se posant à bord des navires ? La chaîne de commandement s'appuyant sur la toute-puissance du capitaine est-elle opératoire quand elle porte sur un objet technique - la machine à vapeur - dont seuls les hommes qui en ont la charge possèdent à la fois les connaissances théoriques et les moyens pratiques d'intervention ? La question des compétences reste centrale, comme le rappelle l'argumentaire erroné du jugement du tribunal civil de Marseille de 1853 à propos de l'explosion de l'Industrie : «[...] le capitaine, qui a la direction du navire, le commandement supérieur, n’a aucune autorité sur la direction des machines [...] ; et qu'en ce qui concerne le service de la machine, ils [les choix] ne relèvent pas du capitaine, et en sont tout à fait indépendans ${ }^{26}$ ".

\section{Du métier à la profession}

Dès l'apparition du bateau à vapeur, les missions du mécanicien de la marine marchande sont claires. L’ordonnance du 17 janvier 1846 les fige dans la réglementation :

de 1857, la Marine s'assure donc plus facilement les services d'un personnel indispensable à son fonctionnement.

25. Duvergier, 1864, p. 433.

26. Cf. note 15 . 
Le mécanicien, sous l'autorité du capitaine, présidera à la mise en feu avant le départ ; il entretiendra toutes les parties de l'appareil moteur ; il s'assurera qu'elles fonctionnent bien, et que les chauffeurs sont en état de bien faire leur service. Pendant le voyage, il dirigera les chauffeurs, et s'occupera de l'appareil moteur et s'occupera constamment de la conduite de la machine.

Ces compétences sont-elles reconnues? Financièrement, sans doute aucun. La rareté de ses capacités techniques et la concurrence, sur le marché du travail, du monde de l'industrie valent au mécanicien d'être le personnel le mieux payé du navire, élément qu'il faut verser au dossier pour expliquer les problèmes de relations hiérarchiques à bord des bâtiments. Ainsi, sur l'Industrie, le mécanicien en chef gagne autour de 4500 francs par an, alors que le capitaine du navire à qui il doit obéir touche un salaire de 4000 francs $^{27}$. S'agit-il d'une pratique habituelle? Apparemment oui pour les petites compagnies, comme le démontre le rôle d'équipage de 1849 de la Ville de Bordeaux, navire de la compagnie marseillaise André \& Abeille, avec un salaire mensuel du premier mécanicien de 387 francs, alors que le capitaine ne reçoit que 200 francs $^{28}$.

Dans la présentation des missions qui sont assignées aux mécaniciens, la particularité du monde maritime apparaît peu. Avec l'homme en charge de la machine, c'est l'atelier qui monte à bord, c'est l'industrie qui gagne la mer. Cette question de compétences exogènes amène à s'interroger sur l'identité du mécanicien de la navigation maritime. Un travailleur peut s'identifier à une entreprise, à un secteur d'activité ou alors à un savoirfaire. Dans le dernier cas, il légitime des valeurs extérieures aux structures qui l'emploient, comme l'illustre parfaitement le monde des mécaniciens de marine, se définissant pendant plusieurs décennies avant tout autour des connaissances et des gestes du forgeron, du chaudronnier et de l'ajusteur, le plus souvent appris dans les écoles d'arts et métiers ${ }^{29}$, qui forment

27. Finidori, Mambrini, 2009, p. 32 (passage consacré aux salaires des membres d'équipage des navires Valéry frères autour de 1850).

28. "État de paiement des salaires dus à l'équipage du paquebot Ville-de-Bordeaux, capitaine Combes, pendant le mois de mai 1849 ", 1930, p. 68. La situation semble avoir été durable car le salaire mensuel du mécanicien en chef est encore de 300 francs et celui du capitaine de 250 francs à Marseille dans la seconde moitié des années 1860 (Enquête parlementaire sur la marine marchande, 1870, p. 180).

29. Bonnefoux et Paris, 1859, p. 463-464. 
l'essentiel des personnels en charge des machines à vapeur dans la France des années 1840-1850. Avant 1846, être mécanicien sur un navire, c'est surtout un métier et non pas une profession maritime. Aucun organisme n'atteste des capacités ou ne délivre de certificat permettant l'exercice à bord des navires. L'affaire de l'Industrie se situe au cour d'une période menant le mécanicien de marine sur la voie de la professionnalisation.

C'est l'ordonnance royale de 1846 qui institue le principe d'une certification des compétences par les pouvoirs publics. L'article 40 exprime cette nouveauté : "nul ne pourra être employé en qualité de mécanicien, s'il ne produit des certificats de capacité délivrés dans les formes qui seront déterminées par notre ministre des travaux publics $»^{30}$. Cette mesure reprend celle qui avait été mise en place trois années plus tôt pour les bateaux de rivière. Les modalités pratiques de la délivrance de ces certificats sont d'ailleurs les mêmes. Le préfet s'appuie sur l'ingénieur - normalement l'ingénieur en chef des Mines - qui s’occupe de la surveillance des bateaux à vapeur dans le département. C'est en effet devant ce dernier que le mécanicien passe son examen, le procès-verbal de l'épreuve étant transmis par la suite à la Préfecture.

Si la loi a été votée, ses effets se font attendre. En 1851, l'année même de l'explosion de l'Industrie, le responsable de l'inscription maritime à Marseille constate en effet que l'ordonnance " est restée sans application » et que la Préfecture des Bouches-du-Rhône ne délivre aucun certificat de capacité aux mécaniciens de la marine marchande locale ${ }^{31}$. La certification des aptitudes du mécanicien ne semble avoir réellement débuté qu'au milieu des années 1850, au moment où l'État fait passer un texte de loi sur les contraventions aux règlements sur les appareils et bateaux à vapeur. Dans cette mesure adoptée le 21 juillet 1856 par le Parlement, une amende de 200 à 2000 francs est instituée pour les propriétaires de navire ayant confié la conduite de l'appareil moteur à un mécanicien ne possédant pas les certificats de capacité nécessaires ${ }^{32}$. La Compagnie Valéry frères ne tardera d'ailleurs pas à être concernée par cette disposition. Elle est en effet condamnée en 1864 pour avoir confié au cours des années 1861-1862 la charge de la machine de son paquebot le Petit Mousse à un dénommé

30. Collection complète des lois, décrets, ordonnances, règlements et avis du Conseil d'État, 1846, p. 41. 31. Enquête parlementaire sur la situation et l'organisation des services de la marine militaire ordonnée par la loi du 31 octobre 1849, 1851, p. 322.

32. Annales des Ponts-et-Chaussées. Lois, décrets, arrêtés et autres actes, $\mathrm{n}^{\circ} 1221,21$ juillet 1856, p. 388. 
Carté, un mécanicien sans titre pour exercer ces fonctions ${ }^{33}$. Le milieu des années 1850 complète également les responsabilités du mécanicien, qui peuvent désormais relever de la justice pénale. La loi du 21 juillet 1856 prévoit des amendes et même des peines d'emprisonnement pour les manquements graves autour de la machine, ceux qui sont susceptibles d'entraîner des explosions de chaudières : de 25 à 250 francs et de trois jours à un mois d'incarcération pour tout mécanicien qui laisse descendre le niveau d'eau dans les générateurs au niveau des conduits de la flamme et de la fumée; de 25 à 500 francs et également de trois jours à un mois pour le fonctionnement d'une machine à une pression non autorisée ou pour avoir volontairement rendu inopérants les appareils de sûretéb ${ }^{34}$.

\section{Réduire l'asymétrie des savoirs sur les appareils à vapeur}

Le fait que le métier de mécanicien de la marine marchande soit devenu une véritable profession, encadrée, surveillée et même éventuellement sanctionnée, ne signifie en rien que les problèmes relationnels avec le capitaine autour des affaires de la machine soient résolus. Afin d'assurer la sécurité sur les navires, de favoriser une conduite efficace des appareils et donner plus de corps à la chaîne d'autorité, le pouvoir législatif doit également se pencher sur les connaissances du capitaine et les faire évoluer pour les mettre en adéquation avec le système technique de la vapeur. L'ordonnance royale du 17 janvier 1846 lance le mouvement. Dans son article 39, il est stipulé que « le capitaine, indépendamment du brevet, soit de capitaine au long cours, soit de maitre de cabotage, [...] devra conformément au mode qui sera déterminé par notre ministre des travaux publics, justifier qu'il possède les connaissances nécessaires pour diriger la marche d'un bâtiment à vapeur et surveiller les opérations du mécanicien ${ }^{35}$ ». Une fois encore, c'est un faux départ. La décision n'est pas immédiatement suivie de faits et le décret impérial du 26 janvier 1857 sur l'admission au commandement des bâtiments du commerce doit de nouveau préciser que « lors des examens pour l'obtention du brevet de capitaine au long cours ou de maître

33. Recueil général des lois et des arrêts, en matière civile, criminelle, administrative et de droit public, 1864, p. 298 et suivantes.

34. Annales des Ponts-et-Chaussées. Lois, décrets, arrêtés et autres actes, n 1221,21 juillet 1856, p. 387.

35. Collection complète des lois, décrets, ordonnances, règlements et avis du Conseil d'État, I846, p. 4I. 
au cabotage, les candidats doivent maîtriser des notions élémentaires sur les machines à vapeur et leurs applications à la navigation ${ }^{36}$ ".

La mesure entre enfin en application, mais reste néanmoins limitée, pour deux raisons. Tout d'abord, elle ne concerne que les nouveaux venus, laissant les choses en l'état pour les capitaines déjà en fonction, qui ne sont appelés ni à repasser leur brevet, ni à compléter leurs connaissances par un moyen ou un autre. Ensuite, la teinture des nouveaux savoirs demandés aux capitaines est légère, de l'aveu même de certains d'entre eux, qui réclament une action bien plus poussée en la matière. La déposition du capitaine nantais Peyrusset devant une commission du Parlement en juin 1870 résume bien les enjeux de la question et les améliorations à apporter sur la formation et les examens professionnels des commandants de navires :

il serait à désirer que les capitaines eussent une parfaite connaissance des machines; car tant qu'un capitaine ne pourra pas contrôler son mécanicien, cela donnera lieu à de graves inconvénients. Si une vergue ou un bas-mât craque, le capitaine ne sait point manier la hache, mais il dira à son charpentier : je veux que vous me fassiez une jumelle de telle ou telle façon, et il sait qu'ainsi jumelée, il peut se servir de sa vergue et continuer sa route jusqu'à sa destination sans relâcher. Il faut qu'il en soit ainsi à l'égard du mécanicien; si une pièce vient à s'échauffer ou casser dans la machine, il faut alors que le capitaine puisse juger par lui-même, si l'avarie peut être réparée avec les ressources du bord, ou si une relâche est nécessaire. Si le capitaine ne peut apprécier l'avarie, il tombera sous la dépendance de son mécanicien, lequel fera relâcher, si c'est son avantage, quand même on aurait pu s'en dispenser ${ }^{37}$.

Juge unique, juge inique... il en va alors de la justice comme de la mécanique marine. Plus de cinq décennies après l'introduction de la vapeur dans la navigation maritime, le principe de décisions collégiales prises par le capitaine et le mécanicien autour des affaires de la machine reste un vœu pieux. L'accident de l'Industrie dans le port de Marseille constitue un observatoire privilégié pour saisir l'évolution du système social des compagnies de

36. Éloy et Guerrand, 1860, p. 58 et suivantes.

37. Enquête parlementaire sur la marine marchande, Paris, 1870, p. 454. 
navigation à vapeur et des relations de travail à bord des steamers au cours du deuxième tiers du XIx ${ }^{e}$ siècle. Il forme dans le même temps un objet dont le droit et la loi se sont emparés pour mieux définir les statuts, les compétences et l'identité des travailleurs embarqués dans les entreprises et devant les tribunaux, que ceux-ci relèvent du civil ou du pénal. Il permet d'entrer au cœur d'une période clé pour le mécanicien de la marine marchande, un moment allant de 1846 à 1864 et durant lequel le métier se transforme en une profession maritime, processus dont on peut cerner les étapes, les rythmes, les freins et les obstacles majeurs. En se focalisant sur le responsable de la machine, le propos retrouve un constat formulé par Ulrich Welke pour la marine marchande à vapeur allemande du XIX ${ }^{\mathrm{e}}$ siècle, autour du temps nécessaire pour que le système technique qui lui est propre arrive à produire "la forme sociale de son accomplissement, assortie à lui " $^{38}$. Trois raisons expliquent cette lenteur dans le cas français. De manière assez classique, la première réside, autour du capitaine et de l'état-major du navire, dans la force de résistance sociale du système de fonctionnement de la navigation à voile ${ }^{39}$. La deuxième se trouve dans l'incapacité des pouvoirs publics français d'appréhender le problème globalement, au lieu de l'envisager avant tout par l'angle, certes primordial, mais très spécifique, de la sécurité des appareils à vapeur. La troisième et dernière explication vient de l'absence de structuration du groupe des mécaniciens de la marine marchande et donc de sa difficulté à peser politiquement et publiquement dans la mise en place des changements.

\section{Sources}

"État de paiement des salaires dus à l'équipage du paquebot Ville-de-Bordeaux, capitaine Combes, pendant le mois de mai 1849 ", Exposition rétrospective de la navigation à vapeur à Marseille, 1818-1900, Le Sémaphore, Marseille, 1930. Bonnefoux Pierre-Marie Joseph et PÂris Edmond, Dictionnaire de marine à voiles et à vapeur. Marine à vapeur, A. Bertrand, Paris, 1859.

BOYer Albert, Étude historique et critique de l'inscription maritime, Paris, 1960.

\footnotetext{
38. Welke, 2014, p. 35.
}

39. Il ne s'agit pas d'opposer un système technique dynamique et sans concurrence (la vapeur) à un autre devenu léthargique et obsolète (la voile). Les travaux de Jean-Louis Lenhof ont bien montré les améliorations apportées aux voiliers durant la seconde moitié du XIX siècle (Lenhof, 2006). Ici, il s'agit avant tout de mettre en avant, pour les équipages, leur fonctionnement et les représentations dont ils font l'objet, l'importance des héritages d'un système technique ancré dans le temps long. 
Autour de l'explosion du navire à vapeur l'Industrie dans le port de Marseille

Collection complète des lois, décrets, ordonnances, règlements et avis du Conseil d'État, Paris, t. XLVI, 1846.

Éloy H. et Guerrand J., Marine marchande. Des capitaines, maîtres et patrons ou traité de leurs droits et obligations au point de vue commercial, civil, administratif et pénal, Guillaumin, Paris, 1860.

Enquête parlementaire sur la marine marchande, Impr. du Journal officiel, Paris, 1870.

Enquête parlementaire sur la situation et l'organisation des services de la marine militaire ordonnée par la loi du 31 octobre 1849, Paris, t. II, 1851.

Journal du Palais. Lois, décrets, règlements et instructions d'intérêt général. Année 1864, Paris, 1865.

PAwlowski Auguste, La marine marchande et l'inscription maritime, A. Challamel, Paris, 1910.

Recueil général des lois et des arrêts, en matière civile, criminelle, administrative et de droit public, Paris, 1864.

\section{Bibliographie}

Alessandri Anne-Marie, "Étude de la Compagnie Valéry et du devenir de sa flotte ", mémoire de maîtrise d'histoire, Université de Provence, 1985, 2 vol.

BARAK Michel, "Quelques tentatives marseillaises d'organisation de la navigation à vapeur (1832-1854) ", Provence historique, n 83, 1971, p. 38-88.

Barillé Claire, Le Roux Thomas, Thébaud-Sorger Marie, "Grenelle, 1794. Secourir, indemniser et soigner les victimes d'une catastrophe industrielle à l'heure révolutionnaire ", Le Mouvement social, n 4, 2014, p. 41-71.

Barzman John, Lenhof Jean-Louis (dir.), "Travail et travailleurs maritimes, $\mathrm{XVIII}^{\mathrm{e}}-\mathrm{XX}^{\mathrm{e}}$ siècle : du métier aux représentations ", Revue d'histoire maritime, $\mathrm{n}^{\circ} 5,2006$.

Berneron-Couvenhes Marie-Françoise, Les messageries maritimes : l'essor d'une grande compagnie de navigation française, 1851-1894, PUPS, Paris, 2007.

Cochard Nicolas, "Les "bouchons gras" dans la ville. Les personnels des machines de navire à vapeur au Havre au XIX ${ }^{\mathrm{e}}$ siècle ", Annales de Normandie, $n^{\circ} 1,2011$, p. 155-179.

Cochard Nicolas, "Les mutations d'une identité professionnelle. Les marins du Havre à l'heure de la modernisation de la navigation au XIX $x^{e}$ siècle ", dans Barzman John, Chevandier Christian (dir.), Dynamique des identités. Travail et organisations, PURH, Mont-Saint-Aignan, 2015, p. 1-25.

Finidori Charles, MAMBrini René, La Compagnie Valéry frères et fils : armateurs cap-corsins, 1840-1883, A. Piazzola, Ajaccio, 2009. 
Fressoz Jean-Baptiste, «L'émergence de la norme technique de sécurité en France vers 1820 ", Le Mouvement social, n 4, 2014, p. 73-89.

JARrige François, Au temps des "tueuses de bras". Les bris de machines à l'aube de l'ère industrielle (1780-1860), PUR, Rennes, 2009.

Landes David, L'Europe technicienne ou le Prométhée libéré. Révolution technique et libre essor industriel en Europe occidentale de 1750 à nos jours, Paris, Gallimard, 1975.

LeNHOF Jean-Louis, "Voile ou vapeur. Étude sur le travail à bord des cargos long-courriers français de la fin du XIx siècle (1880-1920) ", Revue d'histoire maritime, $\mathrm{n}^{\circ}$ 5, mai 2006, p. 99-102.

Lenhof Jean-Louis, Les hommes en mer, de Trafalgar au Vendée Globe, Armand Colin, Paris, 2005.

Welke Ulrich, "Vapeur et travail industriel dans la navigation maritime commerciale au XIX ${ }^{\mathrm{e}}$ siècle ", Revue d'histoire maritime, $\mathrm{n}^{\circ}$ 18, 2014, p. 125-146.

\section{Les auteurs}

Professeur d'histoire contemporaine à Aix-Marseille université, Xavier Daumalin est directeur de l'UMR TELEMMe (AMU-CNRS). Ses recherches portent sur les pollutions industrielles en Méditerranée et sur l'économie marseillaise du Premier Empire à la fin du xIx ${ }^{\mathrm{e}}$ siècle. Il a récemment publié, avec Olivier Raveux, « La marine marchande marseillaise en transition énergétique (1831-1851). Origines et enjeux d'un choix socio-écosystémique ", Cahiers de la Méditerranée, $\mathrm{n}^{\circ}$ 97, 2018, p. 223-239 et, en codirection avec Isabelle Laffont-Schwob, Les calanques industrielles de Marseille et leurs pollutions : une histoire au présent, REF.2C Éditions, Aix-en-Provence, 2016, 336 p. Contact : xavier.daumalin@univ-amu.fr

Chercheur au CNRS, Olivier Raveux est membre de l'UMR TELEMMe (AMU-CNRS). Ses recherches portent sur l'histoire économique et sociale de la Méditerranée à partir du XVII e siècle, principalement dans les domaines de l'histoire maritime, de l'histoire industrielle et des réseaux marchands. Il a récemment publié, en codirection avec Gilbert Buti, Daniel Faget et Solène Rivoal, Moissonner la mer. Économies, sociétés et pratiques halieutiques ( $x v^{e}-x x I^{e}$ siècle), Karthala/MMSH, Paris/ Aix-en-Provence, 2018 et, en codirection avec Gilbert Buti et Luca Lo Basso, Entrepreneurs des mers. Capitaines et mariniers du xvl au xx siècle, Riveneuve éditions, Paris, 2017. Contact : raveux@mmsh.univ-aix.fr 